\title{
Os desafios dos enfermeiros de cuidados paliativos no cenário hospitalar brasileiro: revisão integrativa
}

\author{
The challenges of palliative care nurses in the brazilian hospital scenario: an integrative \\ review
}

\begin{abstract}
Los desafíos de las enfermeras de cuidados paliativos en el entorno hospitalario brasileño: una revisión integradora
\end{abstract}

Laryssa Frauches dos Santos do Espirito Santo ${ }^{1 *}$, Jaqueline Inacio Correia Ferreira ${ }^{1}$, Patrícia

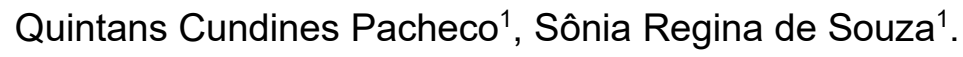

\begin{abstract}
RESUMO
Objetivo: Identificar e analisar os desafios do enfermeiro frente aos pacientes em cuidados paliativos em instituições de saúde no Brasil. Métodos: Trata-se de uma pesquisa qualitativa por meio de uma revisão integrativa de literatura realizada por análise de conteúdo. Esse é um dos métodos de pesquisa utilizados pela Prática Baseada em Evidências (PBE). Ela permite a incorporação das evidências na prática clínica, tendo a finalidade de reunir e sintetizar resultados de pesquisas sobre um delimitado tema/questão, de modo sistemático e ordenado, vindo a contribuir para o aprofundamento do conhecimento do assunto investigado. Resultados: Os estudos foram analisados em sua íntegra de onde emergiu a categoria de análise: A comunicação e o vínculo entre enfermeiros, pacientes e família como elemento essencial no cuidar paliativo. Conclusão: A construção de vínculo na relação enfermeiro paciente mostrou-se uma estratégia para minimizar as dificuldades no cotidiano assistencial. Enfrentar os desafios e buscar a melhoria da qualificação da assistência requer ações institucionais que valorizem a saúde emocional dos enfermeiros que lidam com a dor, o sofrimento e a morte neste cenário.
\end{abstract}

Palavras-chave: Enfermeiros, Cuidados paliativos, Neoplasias.

\begin{abstract}
Objective: The objective was to identify and analyze the challenges faced by nurses in the face of patients in palliative care in health institutions in Brazil. Methods: This is a qualitative research through an integrative literature review performed by content analysis. This is one of the research methods used by Evidence-Based Practice (EPS). It allows the incorporation of evidence in clinical practice, with the purpose of gathering and synthesizing research results on a delimited theme/question, in a systematic and orderly manner, contributing to the deepening of the knowledge of the investigated subject. Results: The studies were analyzed in their entirety from which emerged the category of analysis: Communication and the bond between nurses, patients, and family as an essential element in palliative care. Conclusion: The construction of bonds in the patient nurse relationship proved to be a strategy to minimize the difficulties in daily care. Facing the challenges and seeking improved qualification of care requires institutional actions that value the emotional health of nurses who deal with pain, suffering and death in this scenario.
\end{abstract}

Key words: Nurses, Palliative care, Neoplasms.

1Universidade Federal do Estado do Rio de Janeiro (UNIRIO), Rio de Janeiro - RJ. *E-mail: lary_frauches@hotmail.com SUBMETIDO EM: 7/2019 | ACEITO EM: 8/2019 | PUBLICADO EM: 5/2020 


\section{RESUMEN}

Objetivo: Identificar y analizar los desafíos de las enfermeras a las que se enfrentan los pacientes en cuidados paliativos en instituciones de salud en Brasil. Métodos: Esta es una investigación cualitativa a través de una revisión de la literatura integrativa realizada por el análisis de contenido. Este es uno de los métodos de investigación utilizados por la práctica basada en la evidencia (EPS). Permite la incorporación de evidencia en la práctica clínica, con el propósito de recopilar y sintetizar resultados de investigación sobre un tema/pregunta delimitado, de manera sistemática y ordenada, contribuyendo a la profundización del conocimiento del sujeto investigado. Resultados: Los estudios fueron analizados en su totalidad de los cuales surgió la categoría de análisis: Comunicación y vinculación entre enfermeras, pacientes y familia como elemento esencial en los cuidados paliativos. Conclusión: La construcción de la vinculación en la relación de enfermera paciente resultó ser una estrategia para minimizar las dificultades en la atención diaria. Abordar los desafíos y tratar de mejorar la cualificación de la atención requiere acciones institucionales que valoren la salud emocional de las enfermeras que libren contra el dolor, el sufrimiento y la muerte en este escenario.

Palabras clave: Enfermeros, Cuidados paliativos, Neoplasias.

\section{INTRODUÇÃO}

Esta pesquisa tem como objeto de estudo os desafios dos enfermeiros nos cuidados paliativos no cenário hospitalar brasileiro. O objetivo dos Cuidados Paliativos é oferecer o tratamento eficaz para os sintomas de desconforto que podem acompanhar o paciente, sejam eles causados pela doença ou pelo tratamento (CASA DO CUIDAR, 2019). A palavra paliativo origina-se de Palliare que em latim significa cobrir com manto e Pallium que significa tapar, encobrir, ocultar (SANTANA JCB, et al., 2009).

O cuidado paliativo originou-se no movimento chamado hospice (asilos), nome dado ao local onde era levado as pessoas que estavam em fase terminal. No ano de 1840 na França os hospices eram usados como abrigos para os peregrinos. Em 1967 na Inglaterra, Cecily Saunders e seus colegas deram uma nova origem e filosofia sobre cuidados, este movimento deu um novo conceito do cuidar. Foi neste período que Cecily Saunders fundou St. Christopher's Hospice para abrigar e atender aos pacientes que estavam na fase terminal, pois para ela o enfermo não deveria ser focado apenas no curar e sim até o término de sua vida (MELO AGC e CAMPONERO R, 2009).

Segundo a Organização Mundial de Saúde (OMS), em conceito definido em 1990 e atualizado em 2002, os cuidados paliativos por uma equipe multidisciplinar, tem como objetivo a melhoria da qualidade de vida do paciente e sua família diante de uma doença que ameace a vida desse. Isso implica na prevenção e alívio do sofrimento, da identificação precoce, da avaliação impecável, tratamento da dor e demais sintomas, sejam físicos, sociais, psicológicos e/ou espirituais (WORLD WIDE PALLIATIVE CARE ALLIANCE, 2014).

Os cuidados paliativos também incluem o manejo de problemas emocionais, sociais e espirituais dos pacientes que costumam surgir a partir do adoecimento. A medida que os pacientes se adaptam melhor a nova realidade e se sentem melhor assistidos pela equipe e pela família, eles têm uma melhor qualidade de vida. Eles podem ser fornecidos ao mesmo tempo que tratamentos destinados a curar ou tratar a doença. Pode-se receber cuidados paliativos quando a doença é diagnosticada durante todo o tratamento, durante $o$ seguimento e no final da vida (SIMONI M e SANTOS ML, 2003).

A modalidade terapêutica "cuidados paliativos" surgiu com a finalidade de melhorara qualidade de vida dos acientes e seus familiares ao enfrentares doenças que ameacem a vida, prevenindo e aliviando os sofrimentos físicos, psicossociais e/ou espirituais" (WORLD WIDE PALLIATIVE CARE ALLIANCE, 2014).

Tradicionalmente, o cuidado paliativo é objeto de ação na área oncológica, sendo também utilizado em qualquer situação de finitude da vida, pois $70 \%$ dos pacientes com câncer no mundo vão morrer em decorrência dessa doença, que é acompanhada, normalmente, de sofrimentos, seja do paciente ou de sua família (SIMONI M e SANTOS ML, 2003). 
A criação de unidades para o tratamento da dor e gestão dos pacientes terminais, onde se aplicam os cuidados paliativos ao longo dos anos, tem sido um espaço para a terapêutica com foco no cuidar, entendendo que o cuidado se sobrepõe a certeza da cura. É imperativo que se diminua o sofrimento dando alívio físico e emocional para o ser humano adoecido (MELO AGC e CAMPONERO R, 2009).

Esta visão deve continuar considerando o envelhecimento da população devido ao aumento da expectativa de vida no cenário mundial e suas consequências como: à presença de doenças crônicodegenerativas. Portanto, as equipes de saúde devem estar preparadas para assistir com o propósito de mitigar o sofrimento (McCOUGHLAN M, 2003).

Este estudo se justifica a partir da compreensão de que o cuidado paliativo é uma forma de atenção ativa e abrangente, voltada para os aspectos físicos, emocionais e sociais relacionados à doença que afeta o paciente. Compreender como a doença influencia a vida dos pacientes é um aspecto muito importante na avaliação para cuidados paliativo (FIRMINO F, 2005).

$\mathrm{Na}$ equipe, o profissional enfermeiro costuma ser o elemento de coesão entre a família, o paciente e os médicos. Seu papel é capturar e transferir as necessidades do paciente e suas famílias para a equipe e colaborar efetivamente para o plano terapêutico multidisciplinar (VASCONCELOS EV, et al., 2012). Contudo, O cotidiano dos enfermeiros que cuidam de pacientes em cuidados paliativos é permeado por dificuldades que comprometem a qualidade da assistência prestada.

O enfermeiro nesta área deve avaliar o paciente com um todo analisando suas necessidades. Estar ciente de como o paciente está vivendo a situação é o ponto de partida para o enfermeiro construir o planejamento da assistência com cuidado e atenção. Neste sentido, valorizar as expectativas do paciente e família e exercitar a escuta são ferramentas que auxiliam na melhoria da qualidade do cuidado de enfermagem e estabelecem uma relação de ajuda (FIRMINO F, 2005).

Considerando o exposto, este estudo busca responder a seguinte questão de pesquisa: Quais são os desafios dos enfermeiros no Brasil frente aos pacientes em cuidados paliativos descritos na produção científica?

O objetivo do estudo foi identificar e analisar os desafios do enfermeiro frente aos pacientes em cuidados paliativos em instituições de saúde no Brasil.

\section{MÉTODOS}

Este estudo trata-se de uma revisão integrativa da literatura. Para a etapa de seleção dos artigos foi utilizado como critérios de inclusão os artigos publicados em português, espanhol e inglês, na base de dados Literatura Latino-Americana e do Caribe em Ciências da Saúde (LILACS) e Banco de Dados em Enfermagem (BDENF) no período de 12 de janeiro à 31 de Janeiro de 2018 e que respondiam a questão de pesquisa. Como critério de exclusão, devido à escassez de estudos sobre a temática objeto desse estudo não foi aplicado recorte temporal para essa busca.

Como estratégia de seleção buscou-se artigos que se referiam à pacientes adultos e infantis, portadores de câncer terminal, em intuições de saúde no Brasil por meio dos descritores: enfermeiro; "cuidados paliativos"; "pacientes oncológicos"; "paciente com câncer terminal"; câncer; com o uso do operador booleano "AND". Os critérios de exclusão na seleção dos artigos foram: artigos que tinham como objeto o cuidado paliativo não oncológico, teses, dissertações, monografias e cartas ao editor.

A busca teve como resultados 581 estudos dos quais foram pré-selecionados 227 artigos. Após aplicados os critérios de inclusão e exclusão restaram 9 , sendo que 3 foram excluídos por apresentarem duplicidade entre bases pesquisadas. A amostra final foi composta por 6 artigos, conforme apresentados no Quadro 1.

Para a análise dos artigos utilizou-se a análise de conteúdo proposta por Bardin $L$ (2011) que se constitui nas seguintes fases: pré-análise, exploração do material e tratamento dos resultados, inferência e interpretação. Segue a descrição da seleção dos artigos que compõe a revisão integrativa (Quadro 1). 
Quadro 1 - Estudos da revisão integrativa - Rio de Janeiro-RJ. 2018.

\begin{tabular}{|c|c|c|c|c|}
\hline Artigo & Autor(es)/ano & Base & País & Resultados \\
\hline $\begin{array}{l}\text { Perfil dos profissionais } \\
\text { enfermeiros e suas } \\
\text { relações com pacientes } \\
\text { oncológicos em cuidados } \\
\text { paliativos. }\end{array}$ & $\begin{array}{l}\text { CUNHA AR, et } \\
\text { al., } 2016\end{array}$ & BDENF & BRASIL & $\begin{array}{l}\text { O perfil dos enfermeiros que lidam } \\
\text { com o paciente e suas relações no } \\
\text { processo de morte morrer e a } \\
\text { possibilidade de contribuição na } \\
\text { assistência de enfermagem. }\end{array}$ \\
\hline $\begin{array}{l}\text { Palliative care in highly } \\
\text { complex oncology care: } \\
\text { perceptions of nurses }\end{array}$ & $\begin{array}{l}\text { SILVA MM, et al., } \\
2015\end{array}$ & LILACS & BRASIL & $\begin{array}{l}\text { A necessidade de mudanças } \\
\text { efetivas para atendimento dessas } \\
\text { pessoas, que dependem de } \\
\text { esforço coletivo para qualificar a } \\
\text { prática. }\end{array}$ \\
\hline $\begin{array}{l}\text { Being nurse at a } \\
\text { chemotherapy center } \\
\text { with the death of an } \\
\text { oncologic patient. }\end{array}$ & $\begin{array}{l}\text { LIMA PC, et al., } \\
2014\end{array}$ & LILACS & BRASIL & $\begin{array}{l}\text { O fenômeno apresenta permeado } \\
\text { de dor e em conformidade por } \\
\text { perceberam-se impotentes diante } \\
\text { da finitude da vida, necessitado de } \\
\text { preparo pessoal e apoio } \\
\text { profissional para o convívio com a } \\
\text { morte. }\end{array}$ \\
\hline $\begin{array}{c}\text { Percepção dos } \\
\text { enfermeiros sobre o } \\
\text { significado dos cuidados } \\
\text { paliativos em pacientes } \\
\text { com câncer terminal. }\end{array}$ & $\begin{array}{l}\text { FERNANDES } \\
\text { MA, et al., } 2013\end{array}$ & LILACS & BRASIL & $\begin{array}{l}\text { Evidenciar que os enfermeiros } \\
\text { envolvidos reconheçam a } \\
\text { importância da equipe } \\
\text { multiprofissional, propiciando ao } \\
\text { enfermeiro a reflexão acerca do } \\
\text { uso da comunicação como } \\
\text { elemento essencial do cuidar para } \\
\text { o paciente família em cuidados } \\
\text { paliativos }\end{array}$ \\
\hline $\begin{array}{l}\text { Cuidados paliativos à } \\
\text { criança com câncer }\end{array}$ & $\begin{array}{l}\text { FRANÇA JRFS, } \\
\text { et al., } 2013\end{array}$ & BDENF & BRASIL & $\begin{array}{l}\text { Os cuidados paliativos são um } \\
\text { instrumento eficaz no cuidado com } \\
\text { a criança com câncer, para } \\
\text { promover uma comunicação } \\
\text { autêntica e um vínculo entre ela e } \\
\text { o enfermeiro que pode desenvolver } \\
\text { um processo terapêutico baseado } \\
\text { em valores humanísticos } \\
\text { universais, com benefícios para } \\
\text { ambos }\end{array}$ \\
\hline $\begin{array}{c}\text { A importância dos } \\
\text { cuidados paliativos } \\
\text { prestados pelo } \\
\text { enfermeiro à criança } \\
\text { com câncer em estágio } \\
\text { terminal }\end{array}$ & $\begin{array}{l}\text { BERNARDO } \\
\text { CM, et al., } 2014\end{array}$ & LILACS & BRASIL & $\begin{array}{l}\text { A importância da atuação do } \\
\text { enfermeiro, onde a proximidades } \\
\text { dos vínculos permitirá uma prática } \\
\text { de enfermagem mais efetiva e } \\
\text { consciente entre todos os } \\
\text { envolvidos. }\end{array}$ \\
\hline
\end{tabular}

Fonte: Santo LFSE, et al., 2019.

\section{RESULTADOS E DISCUSSÃO}

Os artigos que compõem esta revisão integrativa são em sua totalidade de pesquisadores enfermeiros. A abordagem qualitativa foi o método de pesquisa utilizado para o desenvolvimento das pesquisas, sendo os estudos subdivididos em: estudos descritivos (2), fenomenológicos (2) e exploratórios (2). Quanto à produção científica por regiões do Brasil houve o predomínio da região nordeste com destaque nos estados da Paraíba 
(2) e de Alagoas com (1) artigo. Os estudos foram analisados em sua íntegra onde emergiu uma categoria de análise: A comunicação e o vínculo entre enfermeiros, pacientes e família como elemento essencial no cuidar paliativo.

O Instituto Nacional de Câncer (INCA) e o Ministério da Saúde (MS), publicaram um manual de cuidados paliativos no ano de 2001, com o intuito de divulgar informações e orientações a profissionais da saúde que prestam assistência a pacientes em cuidados paliativos, tendo como metas promover a finitude da vida de forma digna, com uma terapêutica voltada ao controle sintomático e preservação da qualidade de vida, sem prolongar ou abreviar a sobrevida, sendo indispensável uma abordagem sistemática da equipe multidisciplinar (INCA; MS, 2001).

$\mathrm{Na}$ dimensão do cuidado em saúde, é que todos os profissionais devem saber, "paliar" é quando os cuidados devem ser necessários. Esse tipo de atenção deve ser assegurado, pois vem propiciar um cuidado de qualidade, não importando se é oferecido em uma unidade hospitalar ou em sua residência (SILVA RCF e HORTALE VA, 2006). Mas, a causa de aproximadamente $70 \%$ dos óbitos, ocorrem em hospitais, onde os aspectos culturais, associados aos fatores sociais, como a dificuldade do tratamento e manejos dos sintomas do paciente em seu domicílio, podem ter por causa esses óbitos nesses ambientes (MORITZ RD, et al., 2008).

Os profissionais da saúde são treinados para fazer todo o possível para manter o paciente vivo. Quando eles se veem diante de um indivíduo com doença crônica em fase avançada, que já não responde a qualquer terapêutica curativa, não visando medidas de prolongamento artificial da vida, visam proporcionar o maior conforto possível para que o paciente viva em plenitude tanto quanto puder até o momento da sua morte (BURLÃ C e PY L, 2014).

A Organização Mundial da Saúde (2014) alerta que os países desenvolvidos e em desenvolvimento estão com pessoas vivendo e morrendo sozinhas e cheias de medo, onde suas dores não mitigadas, seus sintomas físicos não vêm sendo controlados e as suas questões psicossociais e/ou espirituais não estão sendo atendidas (BURLÃ C e PY L, 2014).

A assistência paliativa é uma abordagem complexa. Objetiva atender todas as dimensões do ser cuidado e de sua família, priorizando uma equipe multiprofissional. Essa equipe deve ser composta por enfermeiro, psicólogo, médico, assistente social, farmacêutico, nutricionista, fisioterapeuta, fonoaudiólogo, terapeuta ocupacional, dentista e assistente espiritual. Para que esse objetivo seja alcançado, é preciso que o profissional de saúde tenha uma postura reflexiva em relação às práticas de cuidado. Isso porque as unidades hospitalares tenham por prioridade a dignidade do ser humano como totalidade (ACADEMIA NACIONAL DE CUIDADOS PALIATIVOS, 2009; SIQUEIRA KM, et al., 2007).

É preciso que a equipe de saúde participe de todas as fases do cuidado do paciente, implicando em orientá-lo sem coagir e demonstrando os benefícios e desvantagens de cada tratamento, de forma simples a seu nível de compreensão (OLIVEIRA AC e SILVA MJP, 2010).

No estudo intitulado "Cuidados paliativos à criança com câncer', é destacada a importância da comunicação. A dor e o sofrimento causados pela doença podem ser um fator limitante para o diálogo e para a compreensão, ressaltando a importância da comunicação entre o profissional de saúde e o paciente fora de possibilidades terapêuticas, por meio de uma relação interpessoal. $O$ paciente se encontra em uma situação difícil, caracterizada por dor e sofrimento, podendo resultar em angústia e depressão (FRANÇA JRFS, et al., 2005).

Ganhando notoriedade no contexto hospitalar da UTI, o cuidado paliativo ganha notoriedade no contexto hospitalar. Diante dos avanços medicinais, muitos doentes têm suas vidas prolongadas por equipamentos e medicamentos, Isso vem a causar algum tipo de desconforto e/ou sofrimento, requerendo dos profissionais de saúde um atendimento que vai auxiliar na atenuação desses pacientes (SANTOS SILVA PB, et al., 2020). Fora da possibilidade terapêutica, o cuidar de um paciente engloba vários desafios para a equipe de saúde, especialmente a Enfermagem. Esses profissionais são os que mais sentem a realidade do paciente. É deles a responsabilidade de promover o bem estar e o conforto diante de cada paciente e seus familiares (MORAIS EM, et al., 2018). 
A comunicação entre os enfermeiros, familiar e paciente é de grande importância no processo do cuidar, assim cabe ao enfermeiro saber se expressar de forma clara e objetiva, considerando o nível de conhecimento do paciente. A comunicação efetiva facilita o vínculo entre enfermeiro e paciente desenvolvendo assim um processo terapêutico eficaz, trazendo alterações positivas no humor, estabelecendo equilíbrio emocional e promovendo diminuição do medo (FRANÇA JRFS, et al., 2005).

Para a equipe de enfermagem foi considerado um grande desafio o controle dos sentimentos, pois lidar com a perda do paciente traz uma alta carga emocional, como raiva, frustração, impotência, etc. (FRANCO HCP, et al., 2017). Guedes WG e Torres WC (1984) apontam alguns enganos sobre a hospitalização e os aspectos que a rodeiam (doença, morte, cura) que interferem no desenvolvimento pessoal e profissional dos individuas que atuam em hospitais: a concepção de que o hospital é um lugar exclusivamente de cura; mesmo quando a morte é inevitável ninguém deseja morrer; o paciente não quer saber de sua morte: as emoções interferem na objetividade do médico; ha uma impossibilidade de aceitação e preparação para a morte; e, aspectos não técnicos não são importantes para a cura.

Morrer em hospitais, na contemporaneidade, para as equipes de saúde, é um símbolo de fracasso e ineficiência. Esses profissionais, tornaram-se obcecados na tarefa de evitar e impedir a morte, passando como algo a ser combatido a qualquer custo. Como não é possível evitar a morte, esses profissionais passaram a ignorá-la, isolando-a da vida social e afastando-a emocionalmente, espiritualmente e psiquicamente, tanto do paciente em fase terminal quanto de seus familiares (VICENSI MC, 2016).

O Instituto Nacional de Câncer José de Alencar Gomes da Silva (INCA), responsável pela política nacional integrada para o controle e a prevenção do câncer, estimou para o biênio de 2014/2015 a ocorrência de 576 mil casos novos. O câncer de pele do tipo não melanoma (182 mil casos novos) é o mais incidente na população brasileira, seguido pelos tumores da próstata (69 mil), mama feminina (57 mil), cólon e reto (33 mil), pulmão (27 mil), estômago (20 mil) e colo do útero (15 mil), acompanhando o mesmo perfil da magnitude observada para a América Latina (INCA, 2014). Em 2020, a expectativa é do aumento no número de casos novos de câncer. Aproximadamente,15 milhões de pessoas vão apresentar esse diagnóstico, havendo, naturalmente, a necessidade da expansão dos cuidados paliativos (HIGGINSON IJ e EVANS CJ, 2010).

Segundo o IBGE (2009), o aumento da expectativa de vida, o envelhecimento e a mudança do comportamento da densidade demográfica do Brasil projetam estimativas de que o grupo etário de 60 anos ou mais, duplicará em termos absolutos no período de 2000 a 2020, ao passar de 13,9 para 28,3 milhões, elevando-se, em 2050, para 64 milhões.

O envelhecimento populacional constitui um dos maiores desafios para a saúde pública contemporânea, especialmente em países em desenvolvimento, onde esse fenômeno ocorre em ambientes de pobreza e grande desigualdade social. Em 2025, estima-se que, dos dez países do mundo com o maior número de idosos, cinco serão países em desenvolvimento, entre eles, o Brasil (LIMA-COSTA MF, 2003).

No estudo "Percepção dos enfermeiros sobre o significado dos cuidados paliativos em pacientes com câncer terminal", evidenciam-se a importância da comunicação não verbal no cuidado a esse paciente, sendo destacada a importância da observação para o planejamento da assistência ao mesmo, buscando-se a percepção e a compreensão dos sentimentos, assim como a compreensão, a clareza de gestos, expressões, olhares e linguagens simbólicas particulares de quem está próximo do final de vida (LIMA-COSTA MF, 2003).

Dependendo de uma abordagem multidisciplinar, o cuidado paliativo deve produzir uma assistência harmônica e convergente ao paciente sem possibilidades de cura e a sua família. Os integrantes da equipe multiprofissional necessitam ter como meta uma opção de tratamento que se adeque a esses pacientes, tornando-se primordial o resgate da humanização do processo de morrer. Ou seja, a morte sendo vista como parte de um processo da vida (OLIVEIRA AC e SILVA MJP, 2010; BIFULCOVA e IOCHIDA LC, 2009).

Em adaptação da Teoria de Ruesch, o conceito de comunicação terapêutica compreende na habilidade do profissional da saúde em usar todo o seu conhecimento em ajudar o paciente temporariamente incapacitado a conviver com outras pessoas a seu redor, ajustando-se ao que não pode ser mudado e superar os bloqueios à autorrealização, para o enfrentamento de seus problemas, utilizando-o como meio de ajuda terapêutica. 
Esses conhecimentos fundamentais sobre as bases teóricas da comunicação vão ajudar a enfermagem a adquirir entendimento no relacionamento interpessoal e agir positivamente na assistência a esse paciente. $O$ enfermeiro assim, saberá escutar, dar abertura para a realização de questionamentos, falar, ser honesto e demonstrar respeito, ter tempo para a conversa, interesse, etc (STEFANELLI MC, 1993).

A enfermagem deverá identificar os problemas. Isso facilitará o cuidar, permitindo uma melhor assistência ao paciente, especialmente na elaboração de um plano de cuidados, em que o planejamento, o estabelecimento de metas, a seleção das intervenções, a prestação de um atendimento qualificado de enfermagem é necessário (DOENGES ME e MOORHOUSE MF, 2002). A comunicação é relevante na relação terapêutica que se estabelece entre a equipe e o paciente, e tem por finalidade proporcionar confiança, para que se possa alcançar uma relação de ajuda efetiva, no qual o paciente e sua família possam expressar seus temores e angústias (BRITO FM, 2014). Sendo o enfermeiro, o profissional que passa mais tempo com o paciente, especialmente no cenário hospitalar, o estabelecimento de vínculo de respeito, compaixão, solidariedade e de amor ao próximo entre profissional e paciente é muito relevante nesse processo de finitude. Buscar ser o mais empático possível entendendo o contexto da morte que, apesar de carregado de tristeza, saudade e dor, é um processo natural da vida.

Todavia, constatou-se a falta de habilidade e conhecimento por parte de profissionais de enfermagem no que tange à comunicação, muitos profissionais ignoram as técnicas de comunicação se distanciando do contato com esses pacientes. Lidar com sentimentos correlacionados à morte pode ser um desafio. Dessa forma, nota-se a necessidade da capacitação do enfermeiro na assistência ao paciente terminal em cuidados paliativos (BRITO FM, 2014). No estudo "Cuidados paliativos na assistência de alta complexidade em oncologia: percepção de enfermeiro", os autores observaram a deficiência na formação profissional. A qualificação ineficaz do profissional de enfermagem para lidar com o processo de morrer e morte traz repercussões negativas no cotidiano da assistência ao paciente em cuidados paliativos (BERNARDO CM, et al., 2014).

As limitações e a necessidade de lidar com a morte resultam em sensação de impotência no enfermeiro, o que causa sofrimento em consequência do envolvimento com a criança e sua família frente à evolução negativa da doença, podendo resultar em atendimento frio e impessoal (LIMA PC, et al., 2014). Neste sentido, o estudo intitulado "O ser enfermeiro de uma central de quimioterapia frente à morte do paciente oncológico", teve como achado que ao construir vínculo entre o enfermeiro e seu cliente há maior facilidade no tratamento e consequentemente na relação enfermeiro-paciente no processo de fim de vida. Contudo, o estreitamento do vínculo também pode provocar sofrimento ao profissional diante da finitude, criando um grande dilema entre prática profissional e pessoal.

Na perspectiva da atenção integral a questão do câncer, foi instituída a Lei Presidencial $n^{\circ} 12.732$, de 22/11/2012, que dispõe sobre o primeiro tratamento de paciente com neoplasia maligna comprovada e estabelece prazo para seu início. Para tentar diminuir o retardamento do diagnóstico do câncer, a lei estabelece prazo para o paciente ser tratado no SUS. Em seu Art. $2^{\circ}$, assegura que o paciente com neoplasia maligna tem direito ao primeiro tratamento no SUS no prazo de até 60 dias, contados a partir do dia em que for firmado o diagnóstico em laudo patológico ou em prazo menor, conforme a necessidade terapêutica do caso, registrada em prontuário único.

Considerando sua importância epidemiológica e sua magnitude como problema de saúde pública e a necessidade de redução da mortalidade e da incapacidade, em 2013, foi editada a nova Portaria, que trata da política nacional de atenção ao câncer. Guardando coerência com o eixo III do mencionado Plano de Ações Estratégicas para o Enfrentamento das DCNT, a Portaria $n^{\circ} 874$, de 16/05/2013, "institui a Política Nacional para a Prevenção e Controle do Câncer na Rede de Atenção à Saúde das Pessoas com Doenças Crônicas no âmbito do Sistema Único de Saúde (SUS)" (PNPCC-RAS), sendo da responsabilidade do Ministério da Saúde estabelecer diretrizes nacionais para a prevenção e o controle do câncer, estimulando a atenção integral e articulando as diversas ações nos três níveis de gestão do SUS. O objetivo central da política é contribuir para a melhoria da qualidade de vida dos usuários com câncer, por meio de ações de promoção, prevenção, detecção precoce, tratamento oportuno e cuidados paliativos (BRASIL, 2013). 


\section{CONSIDERAÇÕES FINAIS}

Com este estudo verificou-se que os desafios na assistência ao paciente em cuidados paliativos fazem parte do cotidiano dos enfermeiros no cenário hospitalar no Brasil. Ainda há dificuldade em lidar com a morte e a terminalidade. Deficiências na qualificação profissional têm sido referidas pelas pesquisas como consequência direta desta realidade. Faz-se necessário a elaboração de estudos que discutam as estratégias para melhor enfrentamento dos desafios vivenciados pelos enfermeiros neste cenário. Além disso, é preciso que as instituições de saúde que atendam pacientes em cuidados paliativos desenvolvam estratégias para trabalhar os aspectos emocionais de quem está na linha de frente do cuidado.

\section{REFERÊNCIAS}

1. ACADEMIA NACIONAL DE CUIDADOS PALIATIVOS. Manual de Cuidados Paliativos. $1^{a}$ ed. Rio de Janeiro: Diagraphic; 2009.

2. BARDIN L. Análise de Conteúdo. Brasil: Edições 70, 2011.

3. BERNARDO CM, et al. The importance of palliative care provided by the nurse for children with cancer in terminal phase. Revista de Pesquisa e Cuidados Fundamentais (Online), 2014; 6(3): 1221-1230.

4. BRASIL. Ministério da Saúde. Portaria $n^{\circ} 874 / \mathrm{GM}$, de 16 de Maio de 2013. Institui a Política Nacional para a Prevenção e Controle do Câncer na Rede de Atenção à Saúde das Pessoas com Doenças Crônicas no âmbito do Sistema Único de Saúde (SUS). Diário Oficial [da] União, Brasília, DF, Seção 1, p.129-132, mai. 2013.

5. BIFULCO VA e IOCHIDA LC. A formação na graduação dos profissionais de saúde e a educação para o cuidado de pacientes fora de recursos terapêuticos de cura. Revista Brasileira de Educação em Medicina, 2009; 33(1): 92-100.

6. BRITO FM, et al. Communication in death imminence: Perceptions and strategy adopted for humanizing care in nursing. Escola Anna Nery, Revista de Enfermagem, 2014; 18(2): 317-322.

7. BURLÃ C e PY L. Cuidados paliativos: Ciência e proteção ao fim da vida. Cadernos de Saúde Pública, 2014.

8. CASA DO CUIDAR. 2019. Disponível em: https://www.casadocuidar.org.br/

9. CUNHA AR, et al. Perfil dos profissionais enfermeiros e suas relações com pacientes oncológicos em cuidados paliativos. Revista Nursing (São Paulo), 2016; 19(222): 1462-1465.

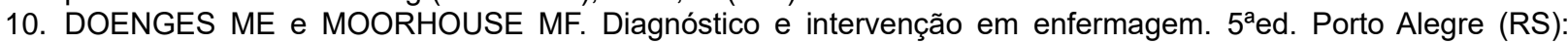
Artmed; 2002.

11. FRANÇA JRFS, et al. Cuidados paliativos à criança com câncer. Revista de Enfermagem da UERJ, 21, 2013; (Esp. 2): 779-784.

12. FRANCO HCP, et al. Papel da enfermagem na equipe de Cuidados Paliativos: A humanização no processo da morte e morrer. Revista Gestão \& Saúde, 2017.

13. FIRMINO F. Pacientes portadores de feridas neoplásicas em serviços de cuidados paliativos: Contribuições para a elaboração de protocolos de intervenções de enfermagem. Revista Brasileira de Cancerologia, 2005; 51(4): 347-359.

14. FERNANDES MA, et al. Percepção dos enfermeiros sobre o significado dos cuidados paliativos em pacientes com câncer terminal. Ciência e Saúde Coletiva, 2013; 18(9): 2589-2596.

15. GUEDES WG e TORRES, WC. A negação da morte e suas implicações na instituição hospitalar. Arquivos Brasileiros de Psicologia, Rio de Janeiro, 1984; 36(4): 102-111.

16. HIGGINSON IJ e EVANS CJ. What is the evidence that palliative care teams improve outcomes for cancer patients and their families, Cancer J, 2010; 16(5): 423-435.

17. IBGE. Instituto Brasileiro de Geografia e Estatística. Indicadores Sociodemográficos e de Saúde no Brasil [internet]. 2009.

18. INSTITUTO NACIONAL DE CÂNCER JOSÉ ALENCAR GOMES DA SILVA (INCA). Estimativa 2014: incidência de câncer no Brasil. Coordenação Geral de Ações Estratégicas, Coordenação de Prevenção e Vigilância. Rio de Janeiro: INCA, 2014.

19. LIMA PC, et al. Being nurse at a chemotherapy center with the death of an oncologic patient. Escola Anna Nery, Revista de Enfermagem, 2014; 18(3): 503-509.

20. LIMA-COSTA MF. Epidemiologia do envelhecimento no Brasil. In: ROUQUAYROL, Z; ALMEIDA FILHO, N. (Org.). Revista Epidemiologia \& Saúde, 6a ed. Rio de Janeiro: MEDSI; 2003.

21. MELO AGC e CAMPONERO R. Cuidados Paliativos - Abordagem contínua e integral. In: SANTOS FS. Cuidados paliativos: Discutindo a vida, a morte e o morrer. São Paulo: Atheneu, 2009.

22. McCOUGHLAN M. A necessidade de cuidados paliativos. Mundo Saúde (Impr.), 2003; 27(1): 6-14.

23. VASCONCELOS EV, et al. Desafios da enfermagem nos cuidados paliativos: Revisão integrativa. Enfermagem em Foco (Brasília), 2012; 3(3): 127-130.

24. MENDES KDS, et al. Revisão integrativa: Método de pesquisa para a incorporação de evidências na saúde e na enfermagem. Texto \& Contexto de Enfermagem, 2008; 17(4): 758-764.

25. MINISTÉRIO DA SAÚDE. Instituto Nacional de Câncer (INCA). Cuidados paliativos oncológicos: Controle de sintomas. Rio de Janeiro (RJ): INCA; 2001. 
26. MORITZ RD, et al. Terminalidade e cuidados paliativos na unidade de terapia intensiva. Revista Brasileira de Terapia Intensiva, 2008; 20(4): 422-428.

27. OLIVEIRAAC e SILVA MJP. Autonomia em cuidados paliativos: Conceitos e percepções de uma equipe de saúde. Acta Paulista de Enfermagem, 2010; 23(2): 212-217.

28. SANTANA JCB, et al. Cuidados paliativos aos pacientes terminais: Percepção da equipe de enfermagem. Bioethikos, 2009; 3(1): 77-86.

29. SANTOS SILVA PB, et al. Os desafios da enfermagem nos cuidados paliativos na unidade de terapia intensiva; 2020.

30. SIMONI M e SANTOS ML. Considerações sobre cuidado paliativo e trabalho hospitalar: Uma abordagem plural sobre o processo de trabalho de enfermagem. Revista de Psicologia da USP, 2003; 14(2): 169-94.

31. SILVA MM, et al. Palliative care in highly complex oncology care: Perceptions of nurses. Escola Anna Nery, Revista de Enfermagem, 2015; 3(19): 460-466.

32. SIQUEIRA KM, et al. O vivenciar a situação de ser com câncer: Alguns desvelamentos. Revista Latino-Americana de Enfermagem, 2007; 15(4): 605-611.

33. STEFANELLI MC. Comunicação com paciente - Teoria e ensino. $2^{a}$ ed. São Paulo (SP): Robe Editorial; 1993.

34. OMS. Organização Mundial de Saúde, World Health Organization. Cancer: palliative care is an essential part of cancer control; 2014.

35. VICENSI MC. Reflexão sobre a morte e o morrer na UTI: A perspectiva do profissional. Revista Bioética, 2016; 24(1).

36. WORLD WIDE PALLIATIVE CARE ALLIANCE. Global atlas of palliative care at the end of life. Geneva: World Health Organization; 2014. 